\title{
Compressed lattice sums arising from the Poisson equation
}

\author{
David H Bailey ${ }^{1}$ and Jonathan M Borwein ${ }^{2 *}$
}

${ }^{*}$ Correspondence:
jonathan.borwein@
newcastle.edu.au
${ }^{2}$ Centre for Computer Assisted
Research Mathematics and Its
Applications (CARMA), University of
Newcastle, Callaghan, NSW 2308,
Australia
Full list of author information is
available at the end of the article

available at the end of the article

\begin{abstract}
Purpose: In recent years attention has been directed to the problem of solving the Poisson equation, either in engineering scenarios (computational) or in regard to crystal structure (theoretical).
\end{abstract}

Methods: In (Bailey et al. in J. Phys. A, Math. Theor. 46:115201, 2013, doi:10.1088/1751-8113/46/11/115201) we studied a class of lattice sums that amount to solutions of Poisson's equation, utilizing some striking connections between these sums and Jacobi $\vartheta$-function values, together with high-precision numerical computations and the PSLQ algorithm to find certain polynomials associated with these sums. We take a similar approach in this study.

Results: We were able to develop new closed forms for certain solutions and to extend such analysis to related lattice sums. We also alluded to results for the compressed sum

$$
\phi_{2}(x, y, d):=\frac{1}{\pi^{2}} \sum_{m, n \in \mathcal{O}} \frac{\cos (\pi m x) \cos (\pi n \sqrt{d y})}{m^{2}+d n^{2}}
$$

where $d>0, x, y$ are real numbers and $\mathcal{O}$ denotes the odd integers. In this paper we first survey the earlier work and then discuss the sum (1) more completely.

Conclusions: As in the previous study, we find some surprisingly simple closed-form evaluations of these sums. In particular, we find that in some cases these sums are given by $1 / \pi \cdot \log A$, where $A$ is an algebraic number. These evaluations suggest that a deep theory interconnects all such summations.

PACS Codes: $02.30 . \mathrm{Lt} ; 02.30 . \mathrm{Mv} ; 02.30 . \mathrm{Nw} ; 41.20 . \mathrm{CV}$

MSC: 06B99; 35J05; $11 Y 40$

Keywords: lattice sums; Poisson equation; experimental mathematics; high-precision computation

\section{Springer}

\section{Background}

In [1], we analyzed various generalized lattice sums [2], which have been studied for many years in the mathematical physics community, for example, in [2-4]. More recently interest was triggered by some intriguing research in image processing techniques [5]. These developments have underscored the need to better understand the underlying theory behind both lattice sums and the associated Poisson potential functions. 
In recent times, attention was directed to the problem of solving the Poisson equation, either in engineering scenarios (computationally, say for image enhancement) or in regard to crystal structure (theoretically). In [1] we thus studied a class of lattice sums that amount to Poisson solutions, namely the $n$-dimensional forms

$$
\phi_{n}\left(r_{1}, \ldots, r_{n}\right)=\frac{1}{\pi^{2}} \sum_{m_{1}, \ldots, m_{n} \text { odd }} \frac{e^{i \pi\left(m_{1} r_{1}+\cdots+m_{n} r_{n}\right)}}{m_{1}^{2}+\cdots+m_{n}^{2}}
$$

By virtue of striking connections with Jacobi $\vartheta$-function values, we were able to develop new closed forms for certain values of the coordinates $r_{k}$ and extend such analysis to similar lattice sums. A primary result was that for rational $x, y$, the natural potential $\phi_{2}(x, y)$ is $\frac{1}{\pi} \log A$ where $A$ is an algebraic number. Various extensions and explicit evaluations were given. We also touched on results for the compressed sum

$$
\phi_{2}(x, y, d):=\frac{1}{\pi^{2}} \sum_{m, n \in \mathbb{O}^{2}} \frac{\cos (\pi m x) \cos (\pi n \sqrt{d} y)}{m^{2}+d n^{2}} .
$$

In this paper we discuss (2) in more detail. More detailed motivation can be found in $[1,5]$.

Such work is made possible by number-theoretical analysis, symbolic computation and experimental mathematics, including extensive numerical computations using up to 25,000-digit arithmetic.

In Section 1.1, we describe, consistent with [5], the underlying equations along with 'natural' Madelung constants and relate them to the classical Madelung constants. In Section 1.2, we produce the solutions $\phi_{n}$ which, especially with $n=2$, provide the central objects of our study. In Section 2.1, we describe rapid methods of evaluating $\phi_{n}$ and add computational details in Section 2.2. In Section 3.1 we discuss closed forms for $\phi_{2}(x, y)$. In Section 3.2 we discuss closed forms for related sums in two dimensions. This leads to the introduction of theta function formalism in the next two subsections and allows us to resolve much else. In Sections 3.5 and 3.6 we are then able to evaluate compressed potential sums. Finally, we further discuss computational matters in Section 3.7.

\subsection{Madelung-type sums}

In a recent treatment of 'natural' Madelung constants [5], it is pointed out that the Poisson equation for an $n$-dimensional point-charge source,

$$
\nabla^{2} \Phi_{n}(\mathbf{r})=-\delta(\mathbf{r}),
$$

gives rise to an electrostatic potential - we call it the bare-charge potential - of the form

$$
\begin{aligned}
& \Phi_{n}(\mathbf{r})=\frac{\Gamma(n / 2-1)}{4 \pi^{n / 2}} \frac{1}{r^{n-2}}=: \frac{C_{n}}{r^{n-2}}, \quad \text { if } n \neq 2, \\
& \Phi_{2}(\mathbf{r})=-\frac{1}{2 \pi} \log r=: C_{2} \log r,
\end{aligned}
$$


where $r:=|\mathbf{r}|$. Since this Poisson solution generally behaves as $r^{2-n}$, the previous work [5] defines a 'natural' Madelung constant $\mathcal{N}_{n}$ as (here, $m:=|\mathbf{m}|$ )

$$
\begin{aligned}
& \mathcal{N}_{n}:=C_{n} \sum_{\mathbf{m} \in \mathbb{Z}^{n}}^{\prime} \frac{(-1)^{\mathbf{1} \cdot \mathbf{m}}}{m^{n-2}}, \quad \text { if } n \neq 2, \\
& \mathcal{N}_{2}:=C_{2} \sum_{\mathbf{m} \in \mathbb{Z}^{n}}^{\prime}(-1)^{\mathbf{1} \cdot \mathbf{m}} \log m,
\end{aligned}
$$

where in cases such as this log sum one must infer an analytic continuation [5], as the literal sum is quite non-convergent. This $\mathcal{N}_{n}$ coincides with the classical Madelung constant

$$
\mathcal{M}_{n}:=\sum_{\mathbf{m} \in \mathbb{Z}^{n}}^{\prime} \frac{(-1)^{\mathbf{1} \cdot \mathbf{m}}}{m}
$$

only for $n=3$ dimensions, in which case $\mathcal{N}_{3}=\frac{1}{4 \pi} \mathcal{M}_{3}$. But in all other dimensions there is no obvious $\mathcal{M}, \mathcal{N}$ relation.

A method for gleaning information about $\mathcal{N}_{n}$ is to contemplate the Poisson equation with a crystal charge source, modeled after $\mathrm{NaCl}$ (salt) in the sense of alternating lattice charges:

$$
\nabla^{2} \phi_{n}(\mathbf{r})=-\sum_{\mathbf{m} \in \mathbb{Z}^{n}}(-1)^{\mathbf{1} \cdot \mathbf{m}} \delta(\mathbf{m}-\mathbf{r}) .
$$

Accordingly - based on the Poisson equation (3) - solutions $\phi_{n}$ can be written in terms of the respective bare-charge functions $\Phi_{n}$ as

$$
\phi_{n}(\mathbf{r})=\sum_{\mathbf{m} \in \mathbb{Z}^{n}}(-1)^{\mathbf{1} \cdot \mathbf{m}} \Phi_{n}(\mathbf{r}-\mathbf{m}) .
$$

\subsection{The crystal solutions $\phi_{n}$}

In [5] it is argued that a solution to (8) is

$$
\phi_{n}(\mathbf{r})=\frac{1}{\pi^{2}} \sum_{\mathbf{m} \in \mathbb{O}^{n}} \frac{\prod_{k=1}^{n} \cos \left(\pi m_{k} r_{k}\right)}{m^{2}}
$$

where $\mathbb{O}$ denotes the odd integers (including negative odds). These $\phi_{n}$ do give the potential within the appropriate $n$-dimensional crystal, in that $\phi_{n}$ vanishes on the surface of the cube $[-1 / 2,1 / 2]^{n}$, as is required via symmetry within an NaCl-type crystal of any dimension thus we have solved the Poisson equation subject to Dirichlet boundary conditions.

To render this representation more explicit and efficient, we could write equivalently

$$
\phi_{n}(\mathbf{r})=\frac{2^{n}}{\pi^{2}} \sum_{m_{1}, \ldots, m_{n}>0, \text { odd }} \frac{\cos \left(\pi m_{1} r_{1}\right) \cdots \cos \left(\pi m_{n} r_{n}\right)}{m_{1}^{2}+\cdots+m_{n}^{2}} .
$$

It is also useful that - due to the symmetry inherent in having odd summation indices - we can cavalierly replace the cosine product in (10) with a simple exponential:

$$
\phi_{n}(\mathbf{r})=\frac{1}{\pi^{2}} \sum_{\mathbf{m} \in \mathbb{O}^{n}} \frac{e^{i \pi \mathbf{m} \cdot \mathbf{r}}}{m^{2}} .
$$


This follows from the simple observation that $\prod \exp \left(\pi i m_{k} r_{k}\right)=\prod\left(\cos \left(\pi m_{k} r_{k}\right)+i \times\right.$ $\left.\sin \left(\pi m_{k} r_{k}\right)\right)$, so when the latter product is expanded out, the appearance of even a single sin term is annihilating due to the bipolarity of every index $m_{k}$.

We observe that the convergence of these conditionally convergent sums is by no means obvious, but that results such as [2, Thm. 8.3 and Thm. 8.5] ensure that

$$
\phi_{n}(\mathbf{r}, s):=\frac{1}{\pi^{2}} \sum_{\mathbf{m} \in \mathbb{O}^{n}} \frac{\prod_{k=1}^{n} \cos \left(\pi m_{k} r_{k}\right)}{m^{2 s}}
$$

is convergent and analytic with abscissa $\sigma_{0}$ for $(n-1) / 4 \leq \sigma_{0} \leq(n-1) / 2$, where $\operatorname{Re}(s)=\sigma$. For the central case herein, summing over increasing spheres is analytic in two dimensions for $\sigma_{0} \leq 23 / 73<1 / 2$ and in three dimensions for $\sigma_{0} \leq 25 / 34<1$, but in general the best estimate we have is $\sigma_{0} \leq n / 2-1$, so for $n \geq 5$, to avoid ambiguity, we work with the analytic continuation of (12) from the region of absolute convergence with $\sigma>n / 2$. Indeed, all our transform methods are effectively doing just that.

\section{Methods}

\subsection{Fast series for $\phi_{n}$}

From previous work [5] we know a computational series

$$
\phi_{n}(\mathbf{r})=\frac{1}{2 \pi} \sum_{\mathbf{R} \in \mathbb{O}^{n-1}} \frac{\sinh \left(\pi R\left(1 / 2-\left|r_{1}\right|\right)\right) \prod_{k=1}^{n-1} \cos \left(\pi R_{k} r_{k+1}\right)}{R \cosh (\pi R / 2)}
$$

suitable for any nonzero vector $\mathbf{r} \in[-1 / 2,1 / 2]^{n}$. The previous work also gives an improvement in the case of $n=2$ dimensions, namely the following form for which the logarithmic singularity at the origin has been siphoned off:

$$
\phi_{2}(x, y)=\frac{1}{4 \pi} \log \frac{\cosh (\pi x)+\cos (\pi y)}{\cosh (\pi x)-\cos (\pi y)}-\frac{2}{\pi} \sum_{\mathbf{m} \in \mathbb{O}^{+}} \frac{\cosh (\pi m x) \cos (\pi m y)}{m\left(1+e^{\pi m}\right)} .
$$

These series, (13) and (14) are valid, respectively, for $r_{1}, x \in[-1,1]$.

For clarification, we give here the $(n=3)$-dimensional case of the fast series:

$$
\phi_{3}(x, y, z)=\frac{2}{\pi} \sum_{p, q>0, \text { odd }} \frac{\sinh \left(\frac{\pi}{2} \sqrt{p^{2}+q^{2}}(1-2|x|)\right) \cos (\pi p y) \cos (\pi q z)}{\sqrt{p^{2}+q^{2}} \cosh \left(\frac{\pi}{2} \sqrt{p^{2}+q^{2}}\right)} .
$$

Though it may not be manifest in this asymmetrical-looking series, it turns out that for any dimension $n$ the $\phi_{n}\left(r_{1}, \ldots, r_{n}\right)$ is invariant under permutations and sign-flips. For example, $\phi_{3}(x, y, z)=\phi_{3}(-y, z,-x)$ and so on. It thus behoves the implementor to consider $x$ - which appears only in the first sum of (15) - to be the largest in magnitude of the three coordinates for optimal convergence. A good numerical test case is the exact evaluation $\phi_{3}(1 / 6,1 / 6,1 / 6)=\frac{\sqrt{3}}{4 \pi}$, which we have confirmed to 500 digits.

\subsection{Computational techniques}

In this study, we employ an experimental scheme similar to that used in [1], as well as in numerous other studies that have been performed. In particular, we compute the key expressions in this study to very high precision, then use the PSLQ algorithm in an attempt 
to recognize the computed numerical values in terms of relatively simple, closed-form expressions. Given an $n$-long input vector $\left(x_{i}\right)$ of real numbers, the PSLQ algorithm finds a nontrivial set of integer coefficients $\left(a_{i}\right)$ such that $a_{1} x_{1}+a_{2} x_{2}+\cdots+a_{n} x_{n}=0$ if it exists (to within the tolerance of the numeric system being used).

Numerous experimental evaluations of minimal polynomials satisfied by $\exp \left(8 \pi \phi_{2}(x, y)\right)$ (the case $d=1$ ), and a detailed description of the underlying computational methodology employed to find these evaluations, are presented in [1]. A similar computational scheme was employed in this study to obtain minimal polynomials satisfied by $\exp \left(8 \pi \sqrt{d} \phi_{2}(x, \sqrt{d} y, d)\right)$. For instance, we recover, among a myriad of other evaluations,

$$
\phi_{2}\left(\frac{1}{3}, \frac{1}{3}, 9\right)=\frac{1}{24 \pi} \log \left(\frac{3+2 \sqrt{3}}{9}\right) .
$$

This computational scheme is as follows:

1. Given $x, y$ and $d$, select a conjectured polynomial degree $m$ and a precision level $P$. We typically set the numeric precision level $P$ somewhat greater than $m^{2}$ digits.

2. Compute $\phi_{2}(x, y \sqrt{d}, d)$ to $P$-digit precision using formula (47). Evaluate the four theta functions indicated using the very rapidly convergent formulas given in [6, p.52] or [7].

3. Generate the $(m+1)$-long vector $\left(1, \alpha, \alpha^{2}, \ldots, \alpha^{m}\right)$, where $\alpha=\exp \left(8 \pi \sqrt{d} \phi_{2}(x, y \sqrt{d}, d)\right)$. Note: we have found that without the eight here, the degree of the resulting polynomial would be eight times as high (but the larger polynomials were in fourth or eighth powers). Given the very rapidly escalating computational cost of higher degrees, many of the results in [1] and herein would not be feasible without this factor.

4. Apply the PSLQ algorithm (we employed the two-level multipair variant of PSLQ for $d$ up to 19 , and the three-level multipair variant for $d \geq 20$ [8]) to find a nontrivial $(m+1)$-long integer vector $A=\left(a_{0}, a_{1}, a_{2}, \ldots, a_{m}\right)$ such that $a_{0}+a_{1} \alpha+a_{2} \alpha^{2}+\cdots+a_{m} \alpha^{m}=0$, if such a vector exists. PSLQ (or one of its variants) either finds a vector $A$, which then is the vector of coefficients of an integer polynomial satisfied by $\alpha$ (certified to the 'epsilon' of the numerical precision used), or else exhausts precision without finding a relation, in which case the algorithm nonetheless provides a lower bound on the Euclidean norm of the coefficients of any possible degree- $m$ integer polynomial $A$ satisfied by $\alpha$.

5. If no numerically significant relation is found, try again with a larger value of $m$ and correspondingly higher precision. If a relation is found, try with somewhat lower $m$, until the minimal $m$ is found that produces a numerically significant relation vector $A$. Here 'numerically significant' means that the relation holds to at least 100 digits beyond the level of precision required to discover it. To obtain greater assurance that the polynomial produced by this process is in fact the minimal polynomial for $\alpha$, use the polynomial factorization facilities in Mathematica or Maple to attempt to factor the resulting polynomial.

\section{Results and discussion}

\subsection{Closed forms for the $\phi_{2}$ potential}

Provably we have the following results which were established by factorization of lattice sums after being empirically discovered by the methods described in the next few sections. 
Theorem 1 ([1]) We have

$$
\begin{aligned}
& \phi_{2}(1 / 3,1 / 3)=\frac{1}{8 \pi} \log (1+2 / \sqrt{3}), \\
& \phi_{2}(1 / 4,1 / 4)=\frac{1}{4 \pi} \log (1+\sqrt{2}), \\
& \phi_{2}(1 / 3,0)=\frac{1}{8 \pi} \log (3+2 \sqrt{3}) .
\end{aligned}
$$

Using the integer relation method PSLQ [9] to hunt for results of the form

$$
\exp \left(\pi \phi_{2}(x, y)\right) \stackrel{?}{=} \alpha
$$

for $\alpha$ algebraic, we may obtain and further simplify many results as follows.

Conjecture 2 ([1]) We have discovered and subsequently proven

$$
\begin{aligned}
& \phi_{2}(1 / 4,0) \stackrel{?}{=} \frac{1}{4 \pi} \log \alpha, \quad \text { where } \frac{\alpha+1 / \alpha}{2}=\sqrt{2}+1, \\
& \phi_{2}(1 / 5,1 / 5) \stackrel{?}{=} \frac{1}{8 \pi} \log (3+2 \sqrt{5}+2 \sqrt{5+2 \sqrt{5}}) \\
& \phi_{2}(1 / 6,1 / 6) \stackrel{?}{=} \frac{1}{4 \pi} \log \gamma, \quad \text { where } \frac{\gamma+1 / \gamma}{2}=\sqrt{3}+1, \\
& \phi_{2}(1 / 3,1 / 6) \stackrel{?}{=} \frac{1}{4 \pi} \log \tau, \quad \text { where } \frac{\tau-1 / \tau}{2}=(2 \sqrt{3}-3)^{1 / 4}, \\
& \phi_{2}(1 / 8,1 / 8) \stackrel{?}{=} \frac{1}{4 \pi} \log \left(\frac{1+\sqrt{2-\sqrt{2}}}{\sqrt[4]{2}-1}\right), \\
& \phi_{2}(1 / 10,1 / 10) \stackrel{?}{=} \frac{1}{4 \pi} \log \mu, \quad \text { where } \frac{\mu+1 / \mu}{2}=2+\sqrt{5}+\sqrt{5+2 \sqrt{5}},
\end{aligned}
$$

where the notation $\stackrel{?}{=}$ indicates that we originally only had experimental (extreme-precision numerical) evidence of an equality.

Such hunts are made entirely practicable by (14). Note that for general $x$ and $y$ we have $\phi_{2}(y, x)=\phi_{2}(x, y)=-\phi_{2}(x, 1-y)$, so we can restrict searches to $1 / 2>x \geq y>0$.

Remark 3 (Algebraicity) In light of our current evidence, we conjectured that for $x, y$ rational,

$$
\phi_{2}(x, y) \stackrel{?}{=} \frac{\log \alpha}{\pi}
$$

for $\alpha$ algebraic. Theorem 5 proved this conjecture. We note that Theorem 5 proves that all values should be algebraic but does not, $a$ priori, establish the precise values we have found. This will be addressed in Section 3.4.

\subsection{Madelung and 'jellium' crystals, and Jacobi $\vartheta$-functions}

We have studied

$$
\phi_{2}(x, y):=\frac{1}{\pi^{2}} \sum_{a, b \in \mathbb{O}} \frac{\cos (\pi a x) \cos (\pi b y)}{a^{2}+b^{2}},
$$


as a 'natural' potential for $n=2$ dimensions in the Madelung problem. There is another interesting series, namely

$$
\begin{aligned}
\psi_{2}(x, y) & :=\frac{1}{4 \pi^{2}} \sum_{a, b \in \mathbb{Z}}^{\prime} \frac{\cos (2 \pi a x) \cos (2 \pi b y)}{a^{2}+b^{2}} \\
& =\frac{1}{\pi^{2}} \sum_{a, b \in \mathbb{E}} \frac{\cos (\pi a x) \cos (\pi b y)}{a^{2}+b^{2}},
\end{aligned}
$$

where $\mathbb{E}$ denotes the even integers.

Now it is explained and illustrated in [5] that this $\psi_{2}$ function is the 'natural' potential for a classical jellium crystal and relates to Wigner sums [2]. This involves a positive charge at every integer lattice point, in a bath - a jelly - of uniform negative charge density. As such, the $\psi$ functions satisfy a Poisson equation but with different source term [5]. Note, importantly, that $\psi_{2}$ satisfies Neumann boundary conditions on the faces of the Delord cube - in contrast with the Dirichlet boundary conditions satisfied in the Madelung case.

Briefly, a fast series for $\psi_{n}$ has been worked out [5] as follows:

$$
\psi_{n}(\mathbf{r})=\frac{1}{12}+\frac{1}{2} r_{1}^{2}-\frac{1}{2}\left|r_{1}\right|+\frac{2^{n-3}}{\pi} \sum_{\mathbf{S} \in \mathbb{Z}^{+(n-1)}} \frac{\cosh \left(\pi S\left(1-2\left|r_{1}\right|\right)\right) \prod_{k=1}^{n-1} \cos \left(2 \pi S_{k} r_{k+1}\right)}{S \sinh (\pi S)},
$$

valid on the Delord $n$-cube, i.e., for $\mathbf{r} \in[-1 / 2,1 / 2]^{n}$.

In the following, we shall also use the general form of the general Jacobi theta functions, defined as in [10] and [6, Sec. 2.6]:

$$
\begin{aligned}
& \vartheta_{1}(z, q)=2 \sum_{n=0}^{\infty}(-1)^{n} q^{(n+1 / 2)^{2}} \sin ((2 n+1) z), \\
& \vartheta_{2}(z, q)=2 \sum_{n=0}^{\infty} q^{(n+1 / 2)^{2}} \cos ((2 n+1) z), \\
& \vartheta_{3}(z, q)=1+2 \sum_{n=0}^{\infty} q^{n^{2}} \cos (2 n z), \\
& \vartheta_{4}(z, q)=1+2 \sum_{n=0}^{\infty}(-1)^{n} q^{n^{2}} \cos (2 n z) .
\end{aligned}
$$

One useful relation is

$$
\vartheta_{4}^{2}(z, q) \vartheta_{4}^{2}(0, q)=\vartheta_{3}^{2}(z, q) \vartheta_{3}^{2}(0, q)-\vartheta_{2}^{2}(z, q) \vartheta_{2}^{2}(0, q)
$$

\subsection{Closed forms for $\psi_{2}$ and $\phi_{2}$}

Using the series (23) for high-precision numerics, it was discovered (see [5, Appendix]) that previous lattice-sum literature had concealed a longtime typographical error for certain two-dimensional sums, and that a valid closed form for $\psi_{2}$ is actually

$$
\psi_{2}(x, y)=\frac{x^{2}}{2}+\frac{1}{4 \pi} \log \left(\frac{\Gamma(1 / 4)}{\sqrt{8 \pi} \Gamma(3 / 4)}\right)-\frac{1}{2 \pi} \log \left|\vartheta_{1}\left(\pi(i x+y), e^{-\pi}\right)\right| .
$$


In the Madelung case, it then became possible to cast $\phi_{2}$ likewise in closed form, namely

$$
\phi_{2}(x, y)=\frac{1}{4 \pi} \log |\alpha(z)|, \quad \text { where } \alpha(z):=\frac{\vartheta_{2}^{2}(z, q) \vartheta_{4}^{2}(z, q)}{\vartheta_{1}^{2}(z, q) \vartheta_{3}^{2}(z, q)}
$$

for $q:=e^{-\pi}, z:=\frac{\pi}{2}(y+i x)$. (See [5] and [1, Appendix II] for details.)

Theorem 4 ([1]) For $z:=\frac{\pi}{2}(y+i x)$,

$$
\phi_{2}(x, y)=\frac{1}{2 \pi} \log \left|\frac{\vartheta_{2}(z, q) \vartheta_{4}(z, q)}{\vartheta_{1}(z, q) \vartheta_{3}(z, q)}\right|=\frac{1}{4 \pi} \log \left|\frac{1-\frac{\lambda(z)}{\sqrt{2}}}{1-\frac{1 / \lambda(z)}{\sqrt{2}}}\right|
$$

and

$$
\psi_{2}(x, y)=-\frac{1}{4 \pi} \log |2 \mu(2 z)(\sqrt{2} \lambda(2 z)-1)|,
$$

where

$$
\lambda(z):=\frac{\vartheta_{4}^{2}\left(z, e^{-\pi}\right)}{\vartheta_{3}^{2}\left(z, e^{-\pi}\right)}=\prod_{n=1}^{\infty} \frac{\left(1-2 \cos (2 z) q^{2 n-1}+q^{4 n-2}\right)^{2}}{\left(1+2 \cos (2 z) q^{2 n-1}+q^{4 n-2}\right)^{2}}
$$

and

$$
\mu(z):=e^{-\pi x^{2} / 2} \frac{\vartheta_{3}^{2}\left(z, e^{-\pi}\right)}{\vartheta_{3}^{2}\left(0, e^{-\pi}\right)}=q^{x^{2} / 2} \prod_{n=1}^{\infty} \frac{\left(1+2 \cos (2 z) q^{2 n-1}+q^{4 n-2}\right)^{2}}{\left(1+q^{2 n-1}\right)^{4}}
$$

with $q:=e^{-\pi}$.

We recall the general $\vartheta$-transform giving for all $z$ with $\operatorname{Re} t>0$

$$
\vartheta_{3-k}\left(\pi z, e^{-t \pi}\right)=\sqrt{1 / t} e^{-\pi z^{2} / t} \vartheta_{3+k}\left(i \pi z / t, e^{-\pi / t}\right)
$$

for $k=-1,0,1$ (while $\left.\vartheta_{1}\left(\pi z, e^{-t \pi}\right)=\sqrt{-1 / t} e^{-\pi z^{2} / t} \vartheta_{1}\left(i \pi z / t, e^{-\pi / t}\right)\right)$. In particular, with $t=1$ we derive that

$$
\vartheta_{3-k}\left(\pi(i x+y), e^{-\pi}\right)=e^{-\pi z^{2}} \vartheta_{3+k}\left(\pi(i y-x), e^{-\pi}\right),
$$

which directly relates $|\mu(\pi(y+i x))|$ and $|\mu(\pi(x+i y))|$ in (31). Let

$$
\kappa(z):=\frac{\vartheta_{2}^{2}\left(z, e^{-\pi}\right)}{\vartheta_{3}^{2}\left(z, e^{-\pi}\right)} .
$$

Then

$$
\kappa(i x+y)+\lambda(i x+y)=\sqrt{2}
$$

or $1-\sqrt{2} \kappa(i x+y)=\sqrt{2} \lambda(i x+y)-1$, and (32) then shows

$$
\lambda(i x+y)=\kappa(-x+i y)=\sqrt{2}-\lambda(-x+i y) .
$$


Likewise (28) is unchanged on replacing $\lambda$ by $\kappa$. Hence, it is equivalent to (20) to prove that for all $z=\frac{\pi}{2}(y+i x)$ with $x, y$ rational $\lambda(z)$ in (30) is algebraic.

Theorem 5 (Algebraic values of $\lambda$ and $\mu$, [1]) For all $z=\frac{\pi}{2}(y+i x)$ with $x, y$ rational, the values of $\lambda(z)$ and $\mu(2 z)$ in (30) are algebraic. It follows that $\phi_{2}(x, y)=\frac{1}{4 \pi} \log \alpha$ with $\alpha$ algebraic. Similarly, $\psi_{2}(x, y)=\frac{1}{4 \pi} \log \beta$ for $\beta$ algebraic.

This type of analysis also works for any singular value $\tau=\sqrt{-d}$ and $q=\exp (2 \pi i \tau)$ and so applies to sums with $n^{2}+d m^{2}$ in the denominator, as we see in the next section.

\subsection{Explicit equations for degree 2,3 and 5}

We illustrate the complexity of $\Omega_{m}(u, v)$, the algebraic polynomial linking the input and output as we move from $u=z$ to $v=m z$, by first considering $m=2$ and $m=3$.

Example $6(\lambda(2 z)$ and $\lambda(3 z))$ As described in [1],

$$
\lambda(2 z)^{1 / 2}=2^{3 / 4} \frac{\vartheta_{4}^{4}\left(z, e^{-\pi}\right)-\vartheta_{1}^{4}\left(z, e^{-\pi}\right)}{\vartheta_{3}^{4}\left(z, e^{-\pi}\right)+\vartheta_{1}^{4}\left(z, e^{-\pi}\right)},
$$

and so letting $\tau(z)=\vartheta_{1}^{2}\left(z, e^{-\pi}\right) / \vartheta_{3}^{2}\left(z, e^{-\pi}\right)$, we obtain

$$
\begin{aligned}
& \tau(z)=\sqrt{2} \lambda(z)-1, \\
& \lambda(2 z)=2^{3 / 2}\left(\frac{\lambda^{2}(z)-\tau^{2}(z)}{1+\tau^{2}(z)}\right)^{2} .
\end{aligned}
$$

Iteration yields $\Omega_{2^{n}}$. From this one may recursively compute $\lambda\left(z / 2^{n}\right)$ from $\lambda(z)$ and watch the tower of radicals grow. Correspondingly,

$$
\lambda(3 z)=2 \frac{(\lambda(2 z) \lambda(z)-\tau(2 z) \tau(z))^{2}}{(1+\tau(2 z) \tau(z))^{2} \lambda(z)},
$$

where $\tau(z), \tau(2 z), \lambda(2 z)$ are given by (38) and (39). After simplification, we obtain

$$
\lambda(3 z)=\lambda(z)\left(\frac{\lambda^{4}(z)-6 \lambda^{2}(z)+6 \sqrt{2} \lambda(z)-3}{3 \lambda^{4}(z)-6 \sqrt{2} \lambda^{3}(z)+6 \lambda^{2}(z)-1}\right)^{2} .
$$

In addition, since we have an algebraic relation $\alpha=\frac{1-\lambda / \sqrt{2}}{1-1 / \lambda / \sqrt{2}}$, this allows us computationally to prove the evaluation of $\phi_{2}(x / 2, y / 2)$ and $\phi_{2}(x / 3, y / 3)$ once $\phi_{2}(x, y)$ is determined as it is for the cases of Theorem 1 .

We may perform the same work inductively for $d=2 n+1$ using $(n+1) z \pm n z$ in the known addition formulas for $\vartheta_{k}[1]$ to obtain $\Omega_{5}$ and so on. The inductive step is

$$
\lambda((2 n+1) z)=\frac{2}{\lambda(z)}\left(\frac{\lambda((n+1) z) \lambda(n z)-\tau((n+1) z) \tau(n z)}{1+\tau((n+1) z) \tau(n z)}\right)^{2} .
$$

Example 7 (Empirical computation of $\Omega_{d}$ ) Given $z$ and $u=\lambda(z), v=\lambda(d z)$, we compute $u^{j} v^{k}$ for $0 \leq j \leq J, 0 \leq k \leq K$ up to degree $J, K$ and look for a relation to precision $D$. This 
is a potential candidate for $\Omega_{d}$. If $\Omega_{d}(\lambda(w), \lambda(d w)) \approx 0$ at a precision significantly greater than $D$ and for various choices of $w$, we can reliably determine $\Omega_{d}$ in this way [1].

Example 8 We conclude this subsection by proving the following empirical evaluation:

$$
\phi_{2}(1 / 5,2 / 5) \stackrel{?}{=} \frac{1}{4 \pi} \log 5^{1 / 4} .
$$

Let $w=\frac{\pi}{10}(1+2 i)$ so that

$$
\phi_{2}(2 / 5,1 / 5)=\frac{\log |\alpha(w)|}{4 \pi} \quad \text { while }-\phi_{2}(2 / 5,1 / 5)=\frac{\log |\alpha(2 w)|}{4 \pi}
$$

since $\phi_{2}(2 / 5,4 / 5)=-\phi_{2}(2 / 5,1 / 5)$. We may - with help from a computer algebra system solve for the stronger requirement that $\alpha(2 w)=-\alpha(w)$ using Example 6. We obtain

$$
\alpha(2 w)=\sqrt{\frac{2 i-1}{5}}
$$

so that $|\alpha(2 w)|=5^{-1 / 4}$ and $|\alpha(w)|=5^{1 / 4}$, as required. To convert this into a proof, we can make an a priori estimate of the degree and length of $\alpha(w)$ - using (39), (41) and (42) while $\lambda(5 w)=1$ - and then perform a high precision computation to show no other algebraic number could approximate the answer well enough. The underlying result we appeal to [6, Exercise 8, p.356] is given next in Theorem 9.

In this particular case, we use $P(\alpha)=\alpha^{4}+2 \alpha^{2}+5$ with $\ell=8, d=4$ and need to confirm that $|P(\alpha)|<5^{D / 4} L^{-3} 8^{-D}$. A very generous estimate of $L<10^{12}$ and $D<10^{3}$ shows it is enough to check $|P(\alpha(w))| \leq 10^{-765}$. This is very easy to confirm. Relaxing to $L<10^{100}$, $D<10^{4}$ requires verifying $|P(\alpha(w))| \leq 10^{-7,584}$. This takes only a little longer.

Recall that the length of a polynomial is the sum of the absolute value of the coefficients.

Theorem 9 (Determining a zero) Suppose $P$ is an integral polynomial of degree $D$ and length L. Suppose that $\alpha$ is algebraic of degree $d$ and length $\ell$. Then either $P(\alpha)=0$ or

$$
|P(\alpha)| \geq \frac{\max \{1,|\alpha|\}^{D}}{L^{d-1} \ell^{D}} .
$$

Example $10(\lambda(5 z))$ Making explicit the recipe for $\lambda(5 z)$, we eventually arrive at

$$
\begin{aligned}
& \lambda(5 z)=\lambda(z) \frac{\left(\lambda^{4}(z)-4 \sqrt{2} \lambda^{3}(z)+14 \lambda^{2}(z)-10 \sqrt{2} \lambda(z)+5\right)^{2}}{\left(5 \lambda^{4}(z)-10 \sqrt{2} \lambda^{3}(z)+14 \lambda^{2}(z)-4 \sqrt{2} \lambda(z)+1\right)^{2}} \\
& \times \frac{\left(\lambda^{8}(z)+4 \sqrt{2} \lambda^{7}(z)-32 \lambda^{6}(z)+36 \sqrt{2} \lambda^{5}(z)-34 \lambda^{4}(z)+4 \sqrt{2} \lambda^{3}(z)+8 \lambda^{2}(z)-4 \sqrt{2} \lambda(z)+1\right)^{2}}{\left(\lambda^{8}(z)-4 \sqrt{2} \lambda^{7}(z)+8 \lambda^{6}(z)+4 \sqrt{2} \lambda^{5}(z)-34 \lambda^{4}(z)+36 \sqrt{2} \lambda^{3}(z)-32 \lambda^{2}(z)+4 \sqrt{2} \lambda(z)+1\right)^{2}} .
\end{aligned}
$$

This shows how generous our estimates were in Example 8.

In similar fashion we can now computationally confirm all of the exact evaluations in Conjecture 2 and the like. For example, we know that $|\alpha(\pi(1 / 3+i / 3))|=\mid 1 / \alpha(\pi / 2(2 / 3+$ 
$2 i / 3)) \mid$. This solves to produce $-\alpha(\pi / 2(1 / 3+i / 3))^{2}=1+2 / 3 \sqrt{3}$ and establishes (16) of Theorem 1 . Now Example 6 can be used to produce $\alpha(\pi / 2(1 / 6+i / 6))$ is as given in Conjecture 2 , and so on.

We now turn to our main focus.

\section{5 'Compressed' potentials}

Yet another solution to the Poisson equation with crystal charge source, for $d>0$, is

$$
\phi_{2}(x, y, d):=\frac{1}{\pi^{2}} \sum_{m, n \in \mathbb{O}^{2}} \frac{\cos (\pi m x) \cos (\pi n \sqrt{d} y)}{m^{2}+d n^{2}} .
$$

This is the potential inside a crystal compressed by $1 / \sqrt{d}$ on the $y$-axis, in the sense that the Delord-cube now becomes the cuboid $\{x, y\} \in[-1 / 2,1 / 2] \times[-1 /(2 \sqrt{d}), 1 /(2 \sqrt{d})]$. Indeed, $\phi_{2}(x, y, d)$ vanishes on the faces of this 2 -cuboid (rectangle) for $d>0$ and integer. This is technically only a compression when $d>1$.

Along the same lines as the analysis of (13), we can posit a fast series

$$
\phi_{2}(x, y, d)=\frac{1}{\pi \sqrt{d}} \sum_{R \in \mathbb{O}^{+}} \frac{\sinh (\pi R \sqrt{d}(1 / 2-|x|)) \cos (\pi R \sqrt{d} y)}{R \cosh (\pi R \sqrt{d} / 2)},
$$

valid on the Delord cuboid, i.e., $x \in[-1 / 2,1 / 2], y \in[-1 /(2 \sqrt{d}), 1 /(2 \sqrt{d})]$. Moreover, the logaccumulation technique of [5, Appendix] applied as with Theorem 4 yields the following.

Theorem 11 ( $\vartheta$-representation for compressed potential) We have

$$
\phi_{2}(x, y, d)=\frac{1}{2 \sqrt{d} \pi} \log \left|\frac{\vartheta_{2}(z, q) \vartheta_{4}(z, q)}{\vartheta_{1}(z, q) \vartheta_{3}(z, q)}\right|
$$

where $q:=\exp (-\pi \sqrt{d})$ and $z:=\frac{1}{2} \pi \sqrt{d}(y+i x)$.

This theorem is consistent with $d=1$ in the sense $\phi_{2}(x, y, 1)=\phi_{2}(x, y)$. In addition, for integers $a, b \geq 1$, we have

$$
a^{2} \phi_{2}\left(a x, a y, \frac{b^{2}}{a^{2}}\right):=\frac{1}{\pi^{2}} \sum_{m \in a \mathbb{Q}, n \in b \mathbb{O}} \frac{\cos (\pi m x) \cos (\pi n y)}{m^{2}+n^{2}} .
$$

We note that there are various trivial evaluations such as $\phi_{2}(x, 1 / 4,4)=0$ for all $x$. Moreover, for each positive rational $d$, there is an analogue of Theorem 5 in which $1 / \sqrt{2}$ is replaced by the $d$ th singular value $k_{d}[2,6]$. Thence, $k_{2}=\sqrt{2}-1, k_{3}=(\sqrt{3}-1) / \sqrt{8}$, and $k_{4}=(\sqrt{2}-1)^{2}$. Precisely, we set

$$
\begin{aligned}
& \lambda_{d}(z):=\frac{\vartheta_{4}^{2}(z, \exp (-\pi \sqrt{d}))}{\vartheta_{3}^{2}(z, \exp (-\pi \sqrt{d}))}, \quad \kappa_{d}(z):=\frac{\vartheta_{2}^{2}(z, \exp (-\pi \sqrt{d}))}{\vartheta_{3}^{2}(z, \exp (-\pi \sqrt{d}))}, \\
& \tau_{d}(z):=\frac{\vartheta_{1}^{2}(z, \exp (-\pi \sqrt{d}))}{\vartheta_{3}^{2}(z, \exp (-\pi \sqrt{d}))}
\end{aligned}
$$


and have (where $k_{d}^{\prime}=\sqrt{1-k_{d}^{2}}$ )

$$
k_{d}^{\prime} \lambda_{d}(z)+k_{d} \kappa_{d}(z)=1, \quad k_{d} \lambda_{d}(z)-k_{d}^{\prime} \kappa_{d}(z)=\tau_{d}(z),
$$

(see [6, Prop. 2.1]). We observe that (47) can be written for all $x, y$ as

$$
\phi_{2}(x, y, d)=\frac{1}{4 \sqrt{d} \pi} \log \left|\frac{1-k_{d}^{\prime} \lambda_{d}(z)}{1-k_{d}^{\prime} / \lambda_{d}(z)}\right|,
$$

on appealing to (49) and (50). Hence we may now study $\lambda_{d}$ exclusively.

Fix an integer $m>1$. The addition formulas for the $\vartheta$ 's as given in [6, Section 2.6]

$$
\begin{aligned}
& \vartheta_{3}(z+w, q) \vartheta_{3}(z-w, q) \vartheta_{3}^{2}(0, q)=\vartheta_{3}^{2}(z, q) \vartheta_{3}^{2}(w, q)+\vartheta_{1}^{2}(z, q) \vartheta_{1}^{2}(w, q), \\
& \begin{aligned}
& \vartheta_{4}(z+w, q) \vartheta_{4}(z-w, q) \vartheta_{4}^{2}(0, q)=\vartheta_{4}^{2}(z, q) \vartheta_{4}^{2}(w, q)-\vartheta_{1}^{2}(z, q) \vartheta_{1}^{2}(w, q), \\
& \vartheta_{1}(z+w, q) \vartheta_{1}(z-w, q) \vartheta_{2}(0, q) \vartheta_{3}(0, q)= \vartheta_{1}(z, q) \vartheta_{4}(z, q) \vartheta_{2}(w, q) \vartheta_{3}(w, q) \\
&+\vartheta_{1}(w, q) \vartheta_{4}(w, q) \vartheta_{2}(z, q) \vartheta_{3}(z, q)
\end{aligned}
\end{aligned}
$$

when replacing $z$ by $(m-1) z$ and $w$ by $z$, and appealing to (50), allow one recursively to write $\lambda_{d}(m z)$ algebraically in terms of $\lambda_{d}(z)$. We first give the equation for $\lambda_{d}(2 z)$.

Example $12\left(\lambda_{d}(2 z)\right)$ As for $\lambda(2 z)$, which is the case $d=1$, we have analytically

$$
\begin{aligned}
& \tau_{d}(z)=\frac{\lambda_{d}(z)-k_{d}^{\prime}}{k_{d}}, \\
& \lambda_{d}(2 z)=k_{d}^{\prime-3}\left(\frac{\lambda_{d}^{2}(z)-\tau_{d}^{2}(z)}{1+\tau_{d}^{2}(z)}\right)^{2} .
\end{aligned}
$$

This becomes

$$
\lambda_{d}(2 z)=k_{d}^{\prime}\left(\frac{1+\lambda_{d}^{2}(z)-2 \lambda_{d}(z) / k_{d}^{\prime}}{1+\lambda_{d}^{2}(z)-2 \lambda_{d}(z) \cdot k_{d}^{\prime}}\right)^{2}
$$

and $\lambda_{d}(0)=k_{d}^{\prime}$.

We turn to $\lambda_{d}(3 z)$.

Example $13\left(\lambda_{d}(3 z)\right)$ As for $\lambda(3 z)$, which is the case $d=1$, we have

$$
\begin{aligned}
& \tau_{d}(z)=\frac{\lambda_{d}(z)-k_{d}^{\prime}}{k_{d}}, \quad \tau_{d}(2 z)=\frac{\lambda_{d}(2 z)-k_{d}^{\prime}}{k_{d}}, \\
& \lambda_{d}(3 z)=\frac{1}{k_{d}^{\prime 2} \lambda_{d}(z)}\left(\frac{\lambda_{d}(z) \lambda_{d}(2 z)-\tau_{d}(z) \tau_{d}(2 z)}{1+\tau_{d}(z) \tau_{d}(2 z)}\right)^{2} .
\end{aligned}
$$

In tandem with (57) this becomes

$$
\lambda_{d}(3 z)=\lambda_{d} \frac{\left(4 \lambda_{d}-3 k_{d}^{\prime}-6 \lambda_{d}^{3} k_{d}^{\prime}+\lambda_{d}^{4} k_{d}^{\prime}+4 \lambda_{d} k_{d}^{\prime 2}\right)^{2}}{\left(4 \lambda_{d}^{3}+k_{d}^{\prime}-6 \lambda_{d}^{2} k_{d}^{\prime}-3 \lambda_{d}^{4} k_{d}^{\prime}+4 \lambda_{d}^{3} k_{d}^{\prime 2}\right)^{2}}
$$

since $\lambda_{d}(0)=k_{d}^{\prime}$. 
This can also be determined by using the methods of Example 7 to obtain the algebraic equation linking $u=\lambda_{d}(z)$ and $v=\lambda_{d}(3 z)$ and then carefully factoring the result - guided by results such as Example 10. It could also have been rigorously proven by the method used for corresponding formula with $d=1$ in [1].

We next exhibit several sample compressed-sum evaluations, of which (62) is the most striking.

Theorem 14 (Some explicit compressed sums)

$$
\begin{aligned}
\phi_{2}\left(\frac{1}{3}, \frac{\sqrt{2}}{3}, 2\right)= & \frac{1}{8 \sqrt{2} \pi} \log (9-6 \sqrt{2}), \\
\phi_{2}\left(\frac{1}{3}, \frac{\sqrt{3}}{3}, 3\right)= & \frac{1}{8 \sqrt{3} \pi} \log \left(\frac{1}{3}\right), \\
\phi_{2}\left(\frac{1}{3}, \frac{1}{3}, 4\right)= & \frac{1}{16 \pi} \log \left(\sqrt{3}(2+\sqrt{3})(\sqrt{2}-\sqrt{3})^{2}\right), \\
\phi_{2}\left(\frac{1}{4}, \frac{1}{4}, 9\right)= & \frac{1}{48 \pi} \log \left(\frac{1}{4}(3+5 \sqrt{3}-4 \sqrt{2})(\sqrt{3}-1)^{3}(1+\sqrt{2})^{2}\right. \\
& \left.\cdot(\sqrt{3}-\sqrt{2})^{2}\right) .
\end{aligned}
$$

Proof Let us first sketch a proof of (62). This requires showing $\alpha=\exp \left(8 \pi \sqrt{3} \psi_{2}(1 \sqrt{3}, 1 / 3\right.$, $d))=1 / 3$. Direct computer algebra using (51) shows that it suffices to prove that $\lambda_{3}(z)=$ $1 / \sqrt{2}+1 / \sqrt{6}$ when $z_{3}:=\pi(1 / 2+i \sqrt{3} / 6)$. Now (60) becomes

$$
\lambda_{3}(3 z)=\frac{\lambda_{3}\left(4 \lambda_{3}-3 k_{3}^{\prime}-6 \lambda_{3}^{2} k_{3}^{\prime}+\lambda_{3}^{4} k_{3}^{\prime}+4 \lambda_{3} k_{3}^{\prime 2}\right)^{2}}{\left(4 \lambda_{3}^{3}+k_{3}^{\prime}-6 \lambda_{3}^{2} k_{3}^{\prime}-3 \lambda_{3}^{4} k_{3}^{\prime}+4 \lambda_{3}^{3} k_{3}^{\prime 2}\right)^{2}} .
$$

However, $3 z$ is in the compressed lattice generated by $1, i / \sqrt{3}$ and so

$$
\lambda_{3}(3 z) \in\left\{0, \infty, k_{3}^{\prime}=\frac{\sqrt{3}+1}{2 \sqrt{2}}, \lambda_{3}\left(i \pi \frac{\sqrt{3}}{2}\right)=\frac{\sqrt{3}}{2} \frac{\sqrt{3}-1}{2 \sqrt{2}}\right\} .
$$

From this, we may deduce that $\lambda_{3}(z)$ is a zero of the denominator of (65), whose only real root is $1 / \sqrt{2}+1 / \sqrt{6}$.

To prove (61), we similarly consider (51) and (60) with $d=2$ so that $k_{2}=\sqrt{2}-1$ and $z_{2}:=\pi(1+i / \sqrt{2}) / 3$ is in the compressed lattice. Indeed, $\lambda_{2}(3 z)=0$, and we now deduce that

$$
\lambda_{2}(z)=\frac{\sqrt{2+2 \sqrt{2}}+i \sqrt{10 \sqrt{2}-14}}{2},
$$

and the result again follows.

To prove (63) requires an application of this method with $d=4$ and $x=1 / 3$ and $y=1 / 6$. We likewise may prove (64) (with $d=9$ ). In this final case, $x=1 / 4$ and $y=1 / 12$. In these cases, we need to appeal to both (59) and (60) at least once.

In general, the polynomial for $\alpha=\exp \left(8 \pi \sqrt{d} \psi_{2}(x, y \sqrt{d}, d)\right)$ with $x, y$ rational can be calculated as we describe in the next section. 


\subsection{Further results for $\phi_{2}(a / c, b / c \sqrt{d}, d)$}

As noted, we anticipated similarly algebraic evaluations for $\phi_{2}(a / c, b / c \sqrt{d}, d)$ for nonnegative integer values of $a, b, c, d$. And, as we will show in more examples in the next section, this is indeed true. All such results can, in principle, be rigorously established by the techniques of Example 7 and Example 8 or indeed Theorem 14, but we will not do this. We do however give the first few polynomials, $P_{d}$, for $\exp \left(8 \pi \phi_{2}(1 / d, 1 / \sqrt{d}, d)\right)$, that is, with $a=b=1, c=d$.

Example 15 (Some explicit compressed polynomials for $\exp \left(8 \pi \sqrt{d} \phi_{2}(1 / d, 1 / \sqrt{d}, d)\right)$ ) We present here the polynomials $P_{d}$ when $3 \leq d \leq 9$.

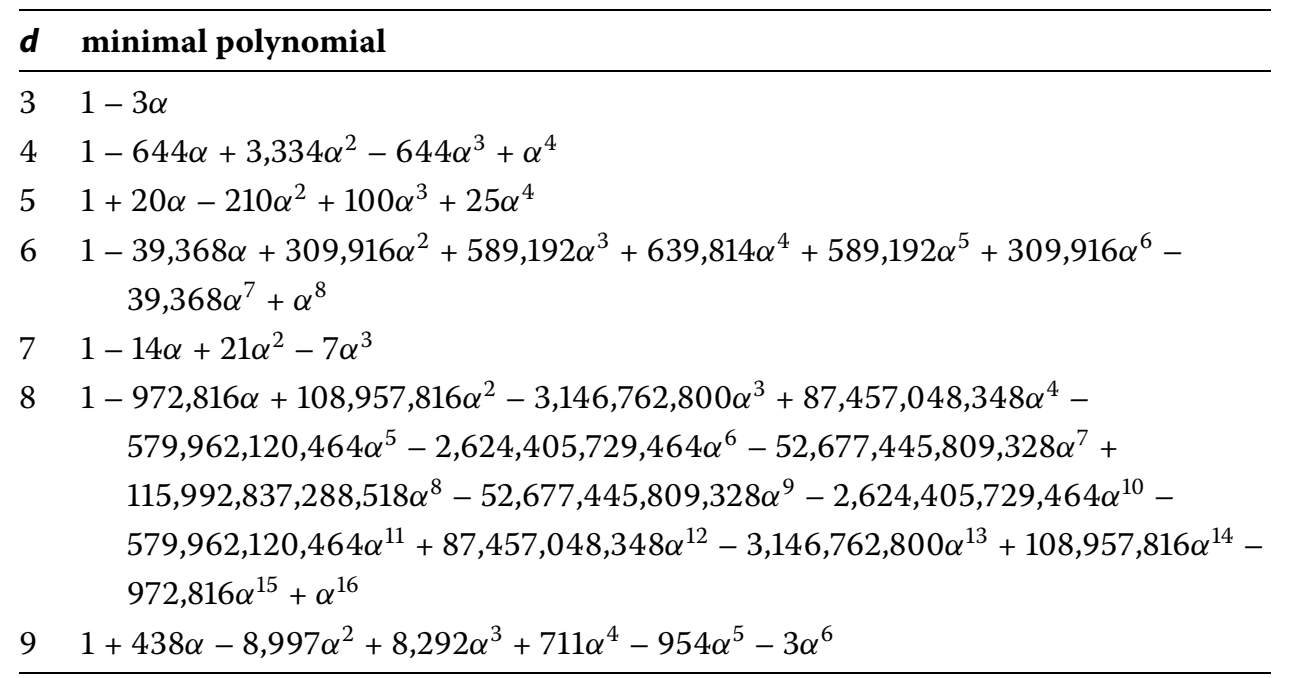

Note that reversing the order of the variables leads to a more intractable computation since $\phi_{2}(1 / \sqrt{d}, 1 / d, d)$ is not of the form covered by Theorem 11 .

\subsection{Computational results}

Results that we have obtained for the special set of cases $\exp \left(8 \pi \sqrt{d} \phi_{2}(1 / d, 1 / \sqrt{d}, d)\right)$, for integers $d$ up to 40, are shown in Table 1. Our computations required up to 25,000-digit precision, and, for large degrees and correspondingly high precision levels, were rather expensive (over 20 processor-hours in some cases). We employed the ARPREC arbitrary precision software [11].

The degree $m(d)$ of the minimal polynomial for $\exp \left(8 \pi \sqrt{d} \phi_{2}(1 / d, 1 / \sqrt{d}, d)\right)$, the number of zeroes $z(d)$ among the minimal polynomial coefficients, the numeric precision level $P$, the run time in seconds $T$, and the approximate base-10 logarithm $M$ of the absolute value of the central coefficient are also shown in the table, together with the ratio $\left(\log _{10} M\right) / m(d)$.

We were not able to obtain relations for all cases $d \leq 40$. Evidently, the precision levels we employed in these cases $(d=32$ and $d=38$ ) were still insufficient to recover the underlying relations; a method to obtain the result for $d=32$ is described in Remark 16 - which works well because $32=4 \times 8$. It is interesting that in this work, as opposed to the $\phi(x, y)$ constants we studied in [1], odd values and prime values of $d$ generally yielded simpler relations than the even instances.

In the earlier study [1], we included Jason Kimberley's observation that the degree $m(d)$ of the minimal polynomial for $\exp \left(8 \pi \phi_{2}(1 / d, 1 / d)\right)$ appeared to be given for odd primes by $m(4 k+1)=(2 k) \cdot(2 k)$ and $m(4 k+3)=(2 k+2) \cdot(2 k+1)$. If one sets $m(2)=1 / 2$, for notational 
Table 1 PSLQ runs to recover minimal polynomials satisfied by $\exp \left(8 \pi \sqrt{d} \phi_{2}(1 / d, 1 / \sqrt{d}, d)\right)$

\begin{tabular}{|c|c|c|c|c|c|}
\hline$d$ & $m(d)$ & $P$ & $T$ & $\log _{10} M$ & $\frac{\log _{10} M}{m(d)}$ \\
\hline 3 & 1 & 200 & 0.00 & & \\
\hline 4 & 4 & 200 & 0.01 & 3.5230 & 0.8807 \\
\hline 5 & 4 & 200 & 0.01 & 2.3222 & 0.5806 \\
\hline 6 & 8 & 200 & 0.02 & 5.8061 & 0.7258 \\
\hline 7 & 3 & 200 & 0.01 & 1.1461 & 0.3820 \\
\hline 8 & 16 & 1,000 & 0.85 & 14.0644 & 0.8790 \\
\hline 9 & 6 & 200 & 0.01 & 3.9187 & 0.6531 \\
\hline 10 & 16 & 1,000 & 0.76 & 12.1262 & 0.7579 \\
\hline 11 & 15 & 1,000 & 0.55 & 7.5230 & 0.5015 \\
\hline 12 & 16 & 1,000 & 0.85 & 15.2686 & 0.9543 \\
\hline 13 & 12 & 1,000 & 0.45 & 9.5690 & 0.7974 \\
\hline 14 & 48 & 4,000 & 100.31 & 38.4539 & 0.8011 \\
\hline 15 & 8 & 200 & 0.03 & 2.1139 & 0.2642 \\
\hline 16 & 32 & 2,000 & 12.51 & 33.7985 & 1.0562 \\
\hline 17 & 32 & 2,000 & 8.93 & 21.9952 & 0.6874 \\
\hline 18 & 24 & 2,000 & 4.32 & 21.1366 & 0.8807 \\
\hline 19 & 27 & 2,000 & 5.27 & 16.8591 & 0.6244 \\
\hline 20 & 64 & 5,000 & 415.78 & 58.8250 & 0.9191 \\
\hline 21 & 24 & 2,000 & 4.52 & 15.6374 & 0.6516 \\
\hline 22 & 40 & 3,000 & 64.33 & 41.4566 & 1.0364 \\
\hline 23 & 33 & 3,000 & 10.92 & 14.4705 & 0.4385 \\
\hline 24 & 64 & 5,000 & 412.59 & 60.3300 & 0.9427 \\
\hline 25 & 20 & 2,000 & 3.77 & 20.0766 & 1.0038 \\
\hline 26 & 144 & 25,000 & $71,680.30$ & 121.91 & 0.8466 \\
\hline 27 & 27 & 2,000 & 5.70 & 18.9234 & 0.7009 \\
\hline 28 & 48 & 4,000 & 131.29 & 58.4901 & 1.2185 \\
\hline 29 & 84 & 6,000 & $1,375.38$ & 60.0921 & 0.7154 \\
\hline 30 & 64 & 5,000 & 557.29 & 56.9952 & 0.8906 \\
\hline 31 & 45 & 4,000 & 38.89 & 19.8425 & 0.4409 \\
\hline 33 & 40 & 4,000 & 81.42 & 32.1363 & 0.8034 \\
\hline 34 & 128 & 21,300 & $45,993.71$ & 123.9012 & 0.9680 \\
\hline 35 & 72 & 12,000 & $1,179.43$ & 41.3569 & 0.5744 \\
\hline 36 & 96 & 12,000 & 95.3311 & 95.3311 & 0.9930 \\
\hline 37 & 36 & 3,000 & 29.11 & 43.5933 & 1.2110 \\
\hline 39 & 48 & 4,000 & 56.33 & 20.7849 & 0.4330 \\
\hline 40 & 128 & 21,300 & & 127.3572 & 0.9950 \\
\hline
\end{tabular}

Here $m(d)$ is the degree, $P$ is the precision level in digits, $T$ is the run time in seconds, and $\log _{10} M$ is the size in digits of the central coefficient. Degrees in bold were obtained by Andrew Mattingly.

convenience, then it appears that for any prime factorization of an integer greater than 2 ,

$$
m\left(\prod_{i=1}^{k} p_{i}^{e_{i}}\right) \stackrel{?}{=} 4^{k-1} \prod_{i=1}^{k} p_{i}^{2\left(e_{i}-1\right)} m\left(p_{i}\right) .
$$

Unfortunately, we have not yet been able to find a similar formula for the minimal polynomial degree in the compressed cases under study in Table 1. We are actively investigating this at the present time.

With regards to the run times listed in Table 1 (given to 0.01 second accuracy), it should be recognized that like all computer run times, particularly in a multicore or multiprocessor environment, they are only repeatable to two or three significant digits. They are listed here only to emphasize the extremely rapid increase in computational cost as the degree $m$ and corresponding precision level $P$ increase. 
Remark 16 (A refined approach) A referee has suggested making more more explicit the relations with elliptic functions. We had opted to limit the theory needed so as to better understand what experimental computation could produce for a wider audience.

(a) The basic quantity $\lambda(z)$, which appears in Theorem 4 , can be written in terms of the Jacobian elliptic function $d n(z)$ [10]. If $z=\pi(y+i x) / 2$, and $k=k_{1}=1 / \sqrt{2}$ as above, then

$$
\lambda(z)=\frac{k}{d n^{2}\left(y K+i x K^{\prime} ; k^{\prime 2}\right)} .
$$

Notice that $K=K^{\prime}$ in this case. All multiplication formulas for $\lambda$ are then essentially special cases of addition formulas for Jacobian elliptic functions. At least in outline this makes it clear that $\lambda(z)$ is algebraic, since elliptic functions evaluated at rational multiples of the periods are always algebraic with respect to $k$. In [12] some closely related lattice sums (essentially linear combinations of some of the lattice sums being considered herein) are evaluated in part by such reasoning.

(b) Similarly, for fixed $0<k<1$, set

$$
f(x):=\frac{1-c n\left(x ; k^{2}\right)}{1+c n\left(x ; k^{2}\right)}, \quad g(u):=4 u \frac{(1+u)^{2}-4 k^{2} u}{\left(1-u^{2}\right)^{2}} .
$$

Then, in terms of the elliptic function $c n[6,10]$, we can write

$$
\phi_{2}(x, y, d)=\frac{1}{4 \pi \sqrt{d}} \log \left|f\left(2 i K_{d}^{\prime} / d, k_{d}\right)\right|
$$

and the corresponding duplication formula for $f$ is $f(2 x)=g(f(x))$. Now the value of $k_{8}$ and the Landen transform [6] yield

$$
k_{32}=\frac{1-\sqrt{1-(5+4 \sqrt{2}-2 \sqrt{14+10 \sqrt{2}})^{2}}}{1+\sqrt{1-(5+4 \sqrt{2}-2 \sqrt{14+10 \sqrt{2}})^{2}}},
$$

[2, Appendix] which makes the minimal polynomial for $k_{32}$ easily expressible

$$
\begin{aligned}
x^{8} & -1,800 x^{7}-12,772 x^{6}-63,800 x^{5}-105,402 x^{4}-63,800 x^{3}-12,772 x^{2}-1,800 x+1 \\
& =0 .
\end{aligned}
$$

Let $\theta_{32}:=f\left(2 i K_{32}^{\prime} / 32, k_{32}\right)$. Observe, much as in earlier examples, that $g\left(g\left(g\left(g\left(\theta_{32}\right)\right)\right)\right)=-1$. We may now obtain an algebraic equation relating $k_{32}, \theta_{32}$ by computing $g(g(g(g(u))))$ while reducing repeatedly using (68); finally, resolvant computations produce the equation for $d=32$ that we failed to find in Table 1 .

\section{Conclusions}

In this study, we have obtained some explicit closed-form evaluations of certain summations that arise as solutions of the Poisson equation, which in turn arises in numerous arenas ranging from engineering to studies of crystal structures. Many of these evaluations were first found experimentally by means of a process we have employed in other settings: computing the relevant expressions to high precision, then applying the PSLQ algorithm 
to recognize the resulting numerical values in closed form. In this case, we computed the constants $\exp \left(8 \pi \sqrt{d} \phi_{2}(1 / d, 1 / \sqrt{d}, d)\right)$, for various integers $d$, to a numeric precision of up to 25,000 digits, then found the integer coefficients of a polynomial that is satisfied by the constant up to a tolerance corresponding to the precision level. We then demonstrated a theoretical framework whereby such experimentally discovered relations can, in many cases, be formally proven.

For further details of the computation of lattice sums and related theta functions the reader is referred to [13-22].

\section{Competing interests}

Neither author has any personal, financial or other competing interests relevant to the topics studied in this paper.

\section{Authors' contributions}

Both authors participated in the research work. DHB performed many of the numerical computations; JMB did much of the theoretical work

\section{Author details}

'Lawrence Berkeley National Laboratory, Berkeley, CA 94720, USA. ${ }^{2}$ Centre for Computer Assisted Research Mathematics and Its Applications (CARMA), University of Newcastle, Callaghan, NSW 2308, Australia.

\section{Acknowledgements}

Dedicated to Professor Hari M Srivastava.

The authors thank many colleagues and the referees for fruitful discussions about lattice sums and theta functions. The first author supported in part by the Director, Office of Computational and Technology Research, Division of Mathematical, Information, and Computational Sciences of the U.S. Department of Energy, under contract number DE-AC02-05CH11231. The second author is supported in part by the Australian Research Council.

\section{Received: 4 December 2012 Accepted: 15 March 2013 Published: 5 April 2013}

\section{References}

1. Bailey, DH, Borwein, JM, Crandall, RE, Zucker, IJ: Lattice sums arising from the Poisson equation. J. Phys. A, Math. Theor. 46, 115201 (2013). doi:10.1088/1751-8113/46/11/115201

2. Borwein, J, Glasser, L, McPhedran, R, Wan, J, Zucker, J: Lattice Sums: Then and Now. Encyclopedia of Mathematics and Its Applications, vol. 150. Cambridge University Press (August 2013, to appear). See also http://www.carma.newcastle. edu.au/jon/LatticeSums/index.html

3. Kanemitsu, S, Tanigawa, Y, Tsukada, H, Yoshimoto, M: Crystal symmetry viewed as zeta symmetry. In: Aoki, T, Kanemitsu, S, Nakahara, M, Ohno, Y (eds.) Zeta Functions, Topology and Quantum Physics, pp. 91-130. Springer, New York (2005) (reprinted 2010)

4. Kanemitsu, S, Tsukada, H: Crystal symmetry viewed as zeta symmetry II. In: Alladi, K, Klauder, JR, Rao, CR (eds.) The Legacy of Alladi Ramakrishnan in the Mathematical Sciences, pp. 275-292. Springer, New York (2010)

5. Crandall, R: The Poisson equation and 'natural' Madelung constants. Preprint (2012)

6. Borwein, JM, Borwein, PB: Pi and the AGM. Wiley, New York (1987)

7. Weisstein, E: Jacobi theta functions. MathWorld, available at http://mathworld.wolfram.com/JacobiThetaFunctions. html

8. Bailey, DH, Broadhurst, DJ: Parallel integer relation detection: techniques and applications. Math. Comput. 70(236), $1719-1736(2000)$

9. Borwein, JM, Bailey, DH: Mathematics by Experiment: Plausible Reasoning in the 21st Century. AK Peters, Wellesley (2004). ISBN:1-56881-136-5. 2nd expanded edn. (2008)

10. Whittaker, ET, Watson, GN: A Course of Modern Analysis, 4th edn. Cambridge University Press, Cambridge (1946)

11. Bailey, DH, Hida, Y, Li, XS, Thompson, B: ARPREC: An arbitrary precision computation package, Sept 2002, available at http://www.davidhbailey.com/dhbpapers/arprec.pdf. The ARPREC software itself is available at http://crd-legacy.lbl. gov/ dhbailey/mpdist

12. Berndt, BC, Lamb, G, Rogers, M: Two-dimensional series evaluations via the elliptic functions of Ramanujan and Jacobi. Ramanujan J. 29(1-3), 185-198 (2012)

13. Crandall, R: Unified algorithms for polylogarithms, L-functions, and zeta variants. In: Algorithmic Reflections: Selected Works. PSIpress, Portland (2011). (Also downloadable at http://www.psipress.com)

14. Forrester, PJ, Glasser, ML: Some new lattice sums including an exact result for the electrostatic potential within the $\mathrm{NaCl}$ lattice. J. Phys. A 15, 911-914 (1982)

15. Glasser, ML: The evaluation of lattice sums. III. Phase modulated sums. J. Math. Phys. 15(188), 188-189 (1974)

16. Glasser, ML, Zucker, IJ: Lattice sums. In: Eyring, H (ed.) Perspectives in Theoretical Chemistry: Advances and Perspectives, vol. 5, pp. 67-139. Academic Press, New York (1980)

17. Zucker, IJ: Exact values for some lattice sums in 2, 4, 6 and 8 dimensions. J. Phys. A 7, 1568-1575 (1974)

18. Zucker, IJ: Functional equations for poly-dimensional zeta functions and the evaluation of Madelung constants. J. Phys. A 9, 499-505 (1976)

19. Zucker, IJ: Some infinite series of exponential and hyperbolic functions. SIAM J. Math. Anal. 15(2), 406-413 (1984)

20. Zucker, IJ: A systematic way of converting infinite series into infinite products. J. Phys. A 20, L13-L17 (1987) 
21. Zucker, IJ: Further relations amongst infinite series and products: II. The evaluation of three-dimensional lattice sums. J. Phys. A 23, 117-132 (1990)

22. Zucker, IJ, MCPhedran, RC: Dirichlet L-series with real and complex characters and their application to solving double sums. Proc. R. Soc. Lond. A 464, 1405-1422 (2008)

doi:10.1186/1687-2770-2013-75

Cite this article as: Bailey and Borwein: Compressed lattice sums arising from the Poisson equation. Boundary Value Problems 2013 2013:75.

Submit your manuscript to a SpringerOpen ${ }^{\circ}$ journal and benefit from:

- Convenient online submission

- Rigorous peer review

- Immediate publication on acceptance

- Open access: articles freely available online

- High visibility within the field

- Retaining the copyright to your article

Submit your next manuscript at $\boldsymbol{s p r i n g e r o p e n . c o m ~}$ 